\title{
La formation des lithalses et leur croissance latérale
} Albert Pissart

\section{Citer ce document / Cite this document :}

Pissart Albert. La formation des lithalses et leur croissance latérale. In: Bulletin de la Classe des sciences, tome 18, $n^{\circ} 7-12$, 2007. pp. 273-294;

doi : https://doi.org/10.3406/barb.2007.28635

https://www.persee.fr/doc/barb_0001-4141_2007_num_18_7_28635

Fichier pdf généré le 05/06/2020 


\begin{abstract}
The lithalsas that occur in Hudsonie, northern Québec, Canada, are modern-day analogues to the features that formed the shallow depressions ('viviers') that occur today on the Hautes Fagnes, Belgium. Observations made in the two regions are complementary. They demonstrate that these periglacial mounds form first by vertical upheaving and later by lateral enlargment.

The main observations in favour of this thesis are : 1) the regular morphology, either cicular or oval, of both actual lithalsas and traces of previous lithalsas, cannot be explained by simple vertical heave ;2) the lateral extension of lithalsas, inferred from relict forms in the Hautes Fagnes, is clearly evident ; 3) the ramparts cannot be explained in terms of simple erosion of the lithalsa surface and subsequent lateral downslope movement of material under gravity to form the ramparts because (a) very low slope angles are involved and (b) thermokarst activity would have been initiated by the erosion ; 4) the oblique inclination of ice lenses observed within a lithalsa in Hudsonie indicates that the freezing plane (i.e. top of permafrost) was lateral, on the sides.

In permafrost regions today, aggradational ice forms by segregation at the permafrost table. If the latter is inclined, the growth of aggradational ice can heave material both upwards and laterally. Therefore, enlargement of a lithalsa, and the preservation of its ramparts following thaw degradation, are thought to be associated with the growth of aggradational ice on the inclined permafrost table that surrounds the mound. This process is called "frost thrusting". The snow accumulations on the border of the lithalses explain the direction of advance of the freezing front. Calculation of the possible pressures developed by the growth of segregated ice supports this thesis.

Figure 14 illustrates how lithalsas are thought to form. This explanation has to be verified in the arctic permafrost region of northern Quebec by excavating sections through actual lithalsas using a portable high pressure water pump.
\end{abstract}

\title{
Résumé
}

La similitude parfaite entre les lithalses de Hudsonie (Québec) et les lithalses qui ont donné naissance, il y a 12000 ans, aux viviers des Hautes Fagnes (Belgique) paraissant établie, les observations faites dans ces deux régions se confortent mutuellement pour montrer que ces buttes périglaciaires après être apparues par un soulèvement vertical du sol, se sont par la suite étendues latéralement.

Les observations principales en faveur de cette thèse sont les suivantes : 1) les formes en plan très régulières, circulaires ou ovales, de nombreuses lithalses actuels et de traces fossiles, sont inexplicables par un simple soulèvement vertical du sol, 2) une extension latérale des lithalses est évidente dans des remparts des formes fossiles des Hautes Fagnes, 3) les déplacements des sédi ments vers les remparts ne peuvent pas être dus seulement à des processus de versants vu la très faible pente de la surface des lithalses et les phénomènes thermokarstiques qu'une telle érosion aurait déclenché, 4) la position oblique en profondeur des lentilles de glace au sein d'une lithalse de Hudsonie est aussi un indice d'une croissance latérale.

Le principal processus responsable de l'élargissement des lithalses et de la formation des remparts qui subsistent après leur fusion consiste en la formation de glace d'accroissement sur la table inclinée du pergélisol en bordure de ces buttes périglaciaires. Cette glace soulève et repousse latéralement la bordure non gelée de la lithalse. Ce processus est appelé «frost thrusting». L'accumulation de neige à la bordure de la lithalse joue un rôle déterminant dans la direction de la progression du gel. Des calculs de la poussée nécessaire pour déplacer les sédiments et aussi l'estimation des pressions développées par la croissance de cette glace de ségrégation confortent la théorie avancée. La figure 14 montre comment se développent vraisemblablement les lithalses dont le tracé en plan est régulier. Cette hypothèse pourra être aisément vérifiée dans l'Arctique en excavant avec une ou des pompes portatives les versants de lithalses actuelles.

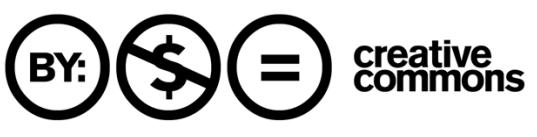




\title{
La formation des lithalses et leur croissance latérale
}

\author{
par Albert Pissart \\ Membre de la Classe
}

\begin{abstract}
The lithalsas that occur in Hudsonie, northern Québec, Canada, are modern-day analogues to the features that formed the shallow depressions ('viviers') that occur today on the Hautes Fagnes, Belgium. Observations made in the two regions are complementary. They demonstrate that these periglacial mounds form first by vertical upheaving and later by lateral enlargment.

The main observations in favour of this thesis are: 1 ) the regular morphology, either cicular or oval, of both actual lithalsas and traces of previous lithalsas, cannot be explained by simple vertical heave; 2) the lateral extension of lithalsas, inferred from relict forms in the Hautes Fagnes, is clearly evident; 3) the ramparts cannot be explained in terms of simple erosion of the lithalsa surface and subsequent lateral downslope movement of material under gravity to form the ramparts because (a) very low slope angles are involved and (b) thermokarst activity would have been initiated by the erosion; 4) the oblique inclination of ice lenses observed within a lithalsa in Hudsonie indicates that the freezing plane (i.e. top of permafrost) was lateral, on the sides.

In permafrost regions today, aggradational ice forms by segregation at the permafrost table. If the latter is inclined, the growth of aggradational ice can heave material both upwards and laterally. Therefore, enlargement of a lithalsa, and the preservation of its ramparts following thaw degradation, are thought to be associated with the growth of aggradational ice on the inclined permafrost table that surrounds the mound. This process is called "frost
\end{abstract}


thrusting". The snow accumulations on the border of the lithalses explain the direction of advance of the freezing front. Calculation of the possible pressures developed by the growth of segregated ice supports this thesis.

Figure 14 illustrates how lithalsas are thought to form. This explanation has to be verified in the arctic permafrost region of northern Quebec by excavating sections through actual lithalsas using a portable high pressure water pump.

\section{Résumé}

La similitude parfaite entre les lithalses de Hudsonie (Québec) et les lithalses qui ont donné naissance, il y a 12000 ans, aux viviers des Hautes Fagnes (Belgique) paraissant établie, les observations faites dans ces deux régions se confortent mutuellement pour montrer que ces buttes périglaciaires après être apparues par un soulèvement vertical du sol, se sont par la suite étendues latéralement.

Les observations principales en faveur de cette thèse sont les suivantes: 1) les formes en plan très régulières, circulaires ou ovales, de nombreuses lithalses actuels et de traces fossiles, sont inexplicables par un simple soulèvement vertical du sol, 2) une extension latérale des lithalses est évidente dans des remparts des formes fossiles des Hautes Fagnes, 3) les déplacements des sédiments vers les remparts ne peuvent pas être dus seulement à des processus de versants vu la très faible pente de la surface des lithalses et les phénomènes thermokarstiques qu'une telle érosion aurait déclenché, 4) la position oblique en profondeur des lentilles de glace au sein d'une lithalse de Hudsonie est aussi un indice d'une croissance latérale.

Le principal processus responsable de l'élargissement des lithalses et de la formation des remparts qui subsistent après leur fusion consiste en la formation de glace d'accroissement sur la table inclinée du pergélisol en bordure de ces buttes périglaciaires. Cette glace soulève et repousse latéralement la bordure non gelée de la lithalse. Ce processus est appelé «frost thrusting». L'accumulation de neige à la bordure de la lithalse joue un rôle déterminant dans la direction de la progression du gel. Des calculs de la poussée nécessaire pour déplacer les sédiments et aussi l'estimation des pressions développées par la croissance de cette glace de ségrégation confortent la théorie avancée. 
La figure 14 montre comment se développent vraisemblablement les lithalses dont le tracé en plan est régulier. Cette hypothèse pourra être aisément vérifiée dans l'Arctique en excavant avec une ou des pompes portatives les versants de lithalses actuelles.

\section{Introduction}

Les viviers des Hautes Fagnes en Belgique sont des traces de lithalses parfaitement conservées, apparues il y a plus de 10.000 ans pendant le Dernier Dryas (Pissart, 2000, 2003). En 1998, nous avons expliqué dans le bulletin de notre classe (Pissart, 1998) ce qu'on savait de ces formes à ce moment. Depuis lors, la connaissance des formes actuelles de Hudsonie s'est affinée et, avec ce que montrent les traces fossiles de Belgique, les faits observés permettent de proposer une explication nouvelle pour rendre compte de la formation des remparts qui subsistent après la fusion de ces buttes périglaciaires.

La thèse de Fabrice Calmels intitulée «Genèse et structure du pergélisol. Etudes des formes périglaciaires de soulèvement au gel au Nunavik (Québec nordique), Canada » était librement disponible sur internet. C'est là que nous l'avons trouvée, lu et relu avec un très grand intérêt. Cette thèse de doctorat a été préparée sous la direction de Michel Allard à la Faculté des Etudes supérieures de l'Université Laval, Québec, dans le cadre du programme de doctorat en sciences géographiques pour l'obtention du grade de Philosophiae Doctor (Ph.D.). Elle a été défendue en 2005 à l'Université de Caen (France).

Cette thèse est consacrée à l'étude de lithalses, palses et plateaux de pergélisol. Beaucoup de données qui y sont rassemblées résultent de l'étude de la lithalse qui a été actuellement le mieux étudiée au monde (figure 1). En effet, des sondages l'ont traversée jusqu'à atteindre la roche en place (gneiss) à une dizaine de mètres de profondeur. Par la suite, par des capteurs de température placés dans ces sondages, l'évolution thermique de cette lithalse a été suivie pendant 5 ans.

Plusieurs sondages réalisés dans cette lithalse ont été effectués grâce à des subsides du «Bundesanstalt für Geowissenschaften und Rohstoffe (BGR, Hannover, Germany)" d'où l'appellation BGR donné à certains des forages. Des articles ont déjà été publiés concernant cette lithalse (Delisle et al., 2003; Buteau et al., 2004; 


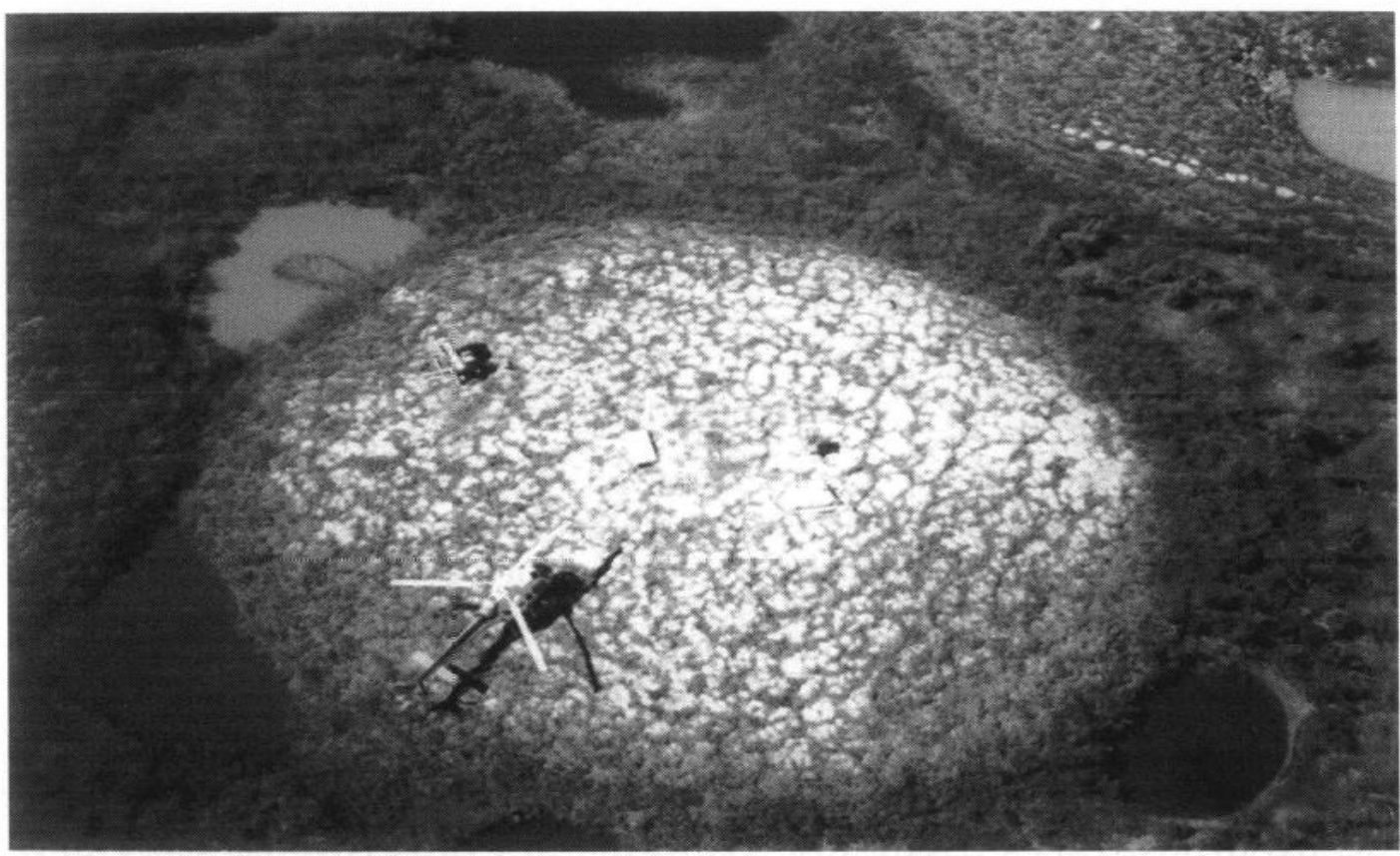

Fig. 1. - La «BGR lithalsa » vue en juillet 2000. Cette forme du Québec septentrional a été étudiée en détails par Calmels (Calmels, thèse fig 3.2, p. 31, 2005). Il en sera longuement question dans le présent article (voir figures 5,8 et 12).

Delisle et Allard, 2003). Des carottes de sondages ont été bien étudiés notamment à l'aide d'une technique employée pour la première fois dans le domaine, en l'occurrence au moyen d'un tomodensimètre à rayon X (Calmels et Allard, 2004) qui a montré parfaitement la distribution de la glace et a permis d'en calculer l'importance.

Les données détaillées dans cette thèse et la comparaison avec les traces de lithalses des Hautes Fagnes indiquent que les lithalses ne développent pas seulement verticalement par soulèvement du sol comme on l'a toujours supposé jusqu'ici, mais qu'elles s'élargissent aussi latéralement en repoussant les matériaux. C'est cette proposition nous développons dans le présent article.

Les chapitres présentés dans la thèse de Calmels, très légèrement modifiés, ont été publiés ou ont été acceptés pour publication dans différentes revues: Calmels et Allard, 2004; Calmels, F. et al., 2005; Calmels, F. et al., 2007a ; Calmels, F. et al., 2007b; Calmels, F. et al., 2007c. 


\section{Les arguments en faveur d'une croissance latérale des lithalses}

\section{DÉPLACEMENT LATÉRAL ENREGISTRÉ DANS LES REMPARTS DES HAUTES Fagnes}

La figure 2 montre une coupe réalisée dans le rempart d'une lithalse des Hautes Fagnes en Belgique (Pissart et Juvigné, 1980; Pissart, 2003) qui établit très clairement que la bordure de la lithalse s'est déplacée latéralement. En effet les formations litées mises en place par ruissellement immédiatement au-dessus de la tourbe indiquent par leur épaisseur, les endroits où se localisait la concavité qui constituait la limite extérieure du rempart. Cette concavité s'est trouvée d'abord en 1, puis par soulèvement de la couche de tourbe et des formations situées en dessous, elle a migré en 2. Par la suite l'arrivée de matériaux sur la pente a déplacé cette concavité de 2 en 3 , un peu au delà de la limite gauche de cette figure 2. La figure 3 montre une tranchée dégagée en bordure de la lithalse ovale, visible sur la photo aérienne de la figure 4. Elle atteste de la même manière un déplacement de la concavité de 1 en 2 sur une distance de l'ordre de $6 \mathrm{~m}$.

Ross Mackay (1979, p. 42) a souligné qu'une différence entre les pingos et les palses (on ne parlait pas des lithalses à ce moment) consistait dans la croissance latérale des secondes alors que les premiers ne se développaient pas latéralement. Comme des tranchées réalisées dans les remparts des Hautes Fagnes (Belgique) dont celle donnée sur la figure 2 , montraient clairement une telle

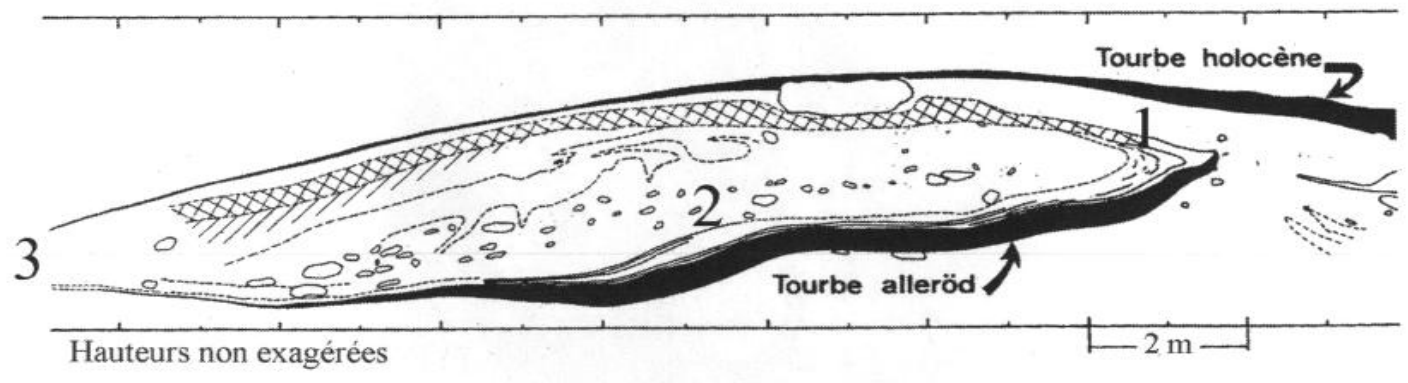

Fig. 2. - Coupe au travers du rempart d'une lithalse des Hautes Fagnes publiée en 1980 par Pissart et Juvigné et reproduite dans Pissart, 2000 et 2003. La couche de tourbe déposée avant la croissance de la lithalse a permis de donner son âge. Les épaississements des couches ruisselées permettent d'établir que la concavité extérieure du rempart a été d'abord en 1 , puis en 2 et enfin, en 3 (ce chiffre devrait être un peu plus à droite, à la rupture de pente limitant le rempart). Le déplacement de 1 en 2 résulte de l'élargissement de la lithalse par poussée profonde, le passage de 2 en 3 est le résultat de l'accumulation de dépôts de pente. 


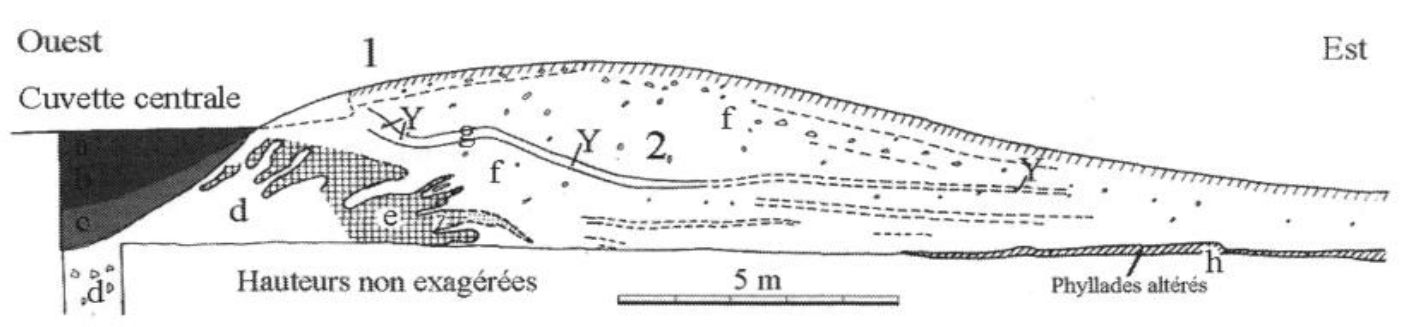

Fig. 3. - Coupe réalisée dans le rempart de la trace de lithalse $\mathrm{X}$ de la figure 4 (Hautes Fagnes, Belgique). L'étude de cette tranchée a été publiée en 1974 (Bastin et al., 1974).

Légende: $a$, tourbe noire; $b$, gyttja; $c$, limon argileux bleu; $d$, limon argileux avec blocs de quartzite; $e$, couche de couleur légèrement différente; $f$, complexe de limon avec débris de quartzite; $g$, couche limoneuse de couleur légèrement différente pratiquement sans cailloux et avec une teneur en carbone élevée qui correspond à la surface du sol avant la croissance de la lithalse; $h$, phyllades très altérés en place. En Y dans la couche g: tephra de l'éruption du Laacher See. La concavité du rempart a été marquée dans la couche g, d'abord en 1, puis en 2.

croissance latérale, nous en avons tiré argument pour défendre que ces dépressions fermées entourées de remparts n'étaient pas des traces de pingos (Pissart, 1980). Cette croissance latérale n'est toutefois enregistrée ici dans la première coupe (figure 2) que sur $15 \mathrm{~m}$ de distance. Le déplacement latéral de 1 en 2 sur une distance de $7 \mathrm{~m}$ est dû à une poussée des formations sous la tourbe; pour les $8 \mathrm{~m}$ suivants, elle résulte de l'accumulation de dépôts de pente en bordure de la lithalse.

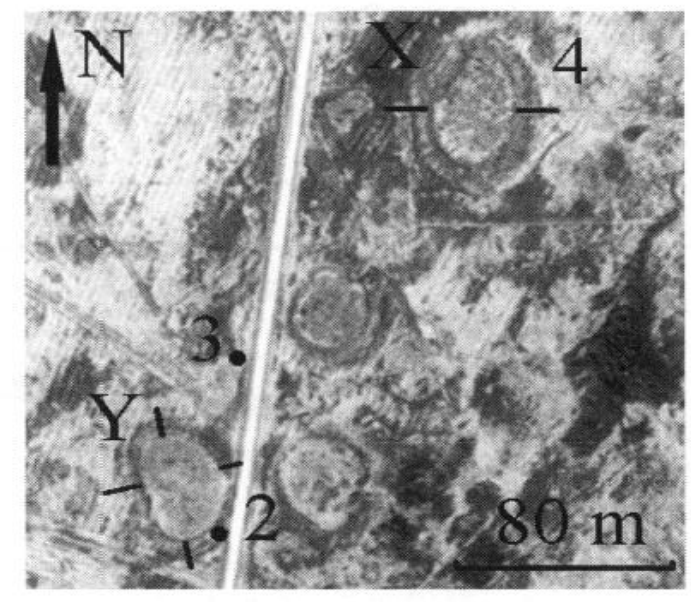

Fig. 4. - Vue aérienne de traces de lithalses ovales au lieu dit la Brackvenn (Hautes Fagnes, Belgique). La forme où la tranchée de la figure 3 a été réalisée, est indiquée par la lettre $X$. Le profil topographique de cette même forme $X$ est donné sur la figure 7. Les deux profils de la lithalse $Y$ sont rassemblés sur la figure 9. Les traits de part et d'autre des remparts donnent l'orientation des profils. La localisation des sondages 2 et 3 est indiquée par ces deux chiffres. En 4, le profil de la figure 3 montre des phyllades en place à $2 \mathrm{~m}$ de profondeur. 
La coupe de la figure 3 (Bastin et al., 1974) montre que $23 \% \mathrm{du}$ rempart de cette trace de lithalse résultent du déplacement de matériaux sous la surface initiale du sol pendant la croissance de la lithalse et que $77 \%$ du rempart proviennent de processus de versant sur les pentes de la lithalse. Pour la coupe de la Konnerzvenn (figure 2), 38\% du rempart sont dus basculement des couches profondes et $62 \%$ aux processus de versants. La déformation des matériaux profonds, sous la surface originelle, ne peut pas résulter d'un simple soulèvement du sol comme nous l'avons supposé en 1980 mais indique l'existence d'un déplacement latéral des sédiments.

\section{FORME RÉGULIÈRE CIRCULAIRE OU OVALE DES LITHALSES}

Certaines traces de lithalses des Hautes Fagnes sont, en plan, parfaitement circulaires ou ovales (figure 4) comme d'ailleurs beaucoup de lithalses de Hudsonie (figure 5) et notamment celle étudiée par F.Calmels (figure 1). A notre connaissance, cette disposition très régulière, circulaire ou ovale, n'a jamais attiré l'attention et aucune explication n'a été avancée pour en rendre compte. Les lithalses diffèrent ainsi nettement des «permafrost plateaus» qui ne donnent pas de remparts après leur fusion dont un exemple a été bien décrit par Allard et al. (1996).

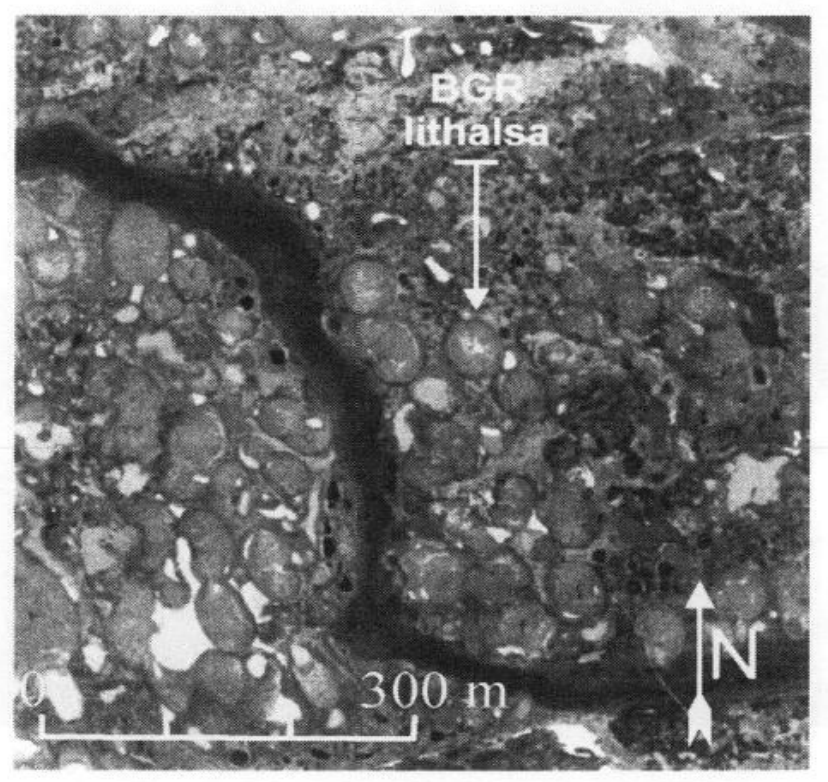

Fig. 5. - Photo aérienne verticale prise en 1957 du champ de lithalses de Hudsonie où se trouve la «BGR lithalsa » de $50 \mathrm{~m}$ de diamètre étudiée par Calmels (Calmels, thèse, figure 5.3, p. 90). 
Des remparts circulaires réguliers vont de soi pour des traces de pingos (glace d'injection) donnant des buttes élevées approximativement coniques sur les pentes desquelles les matériaux descendent par des processus de versant. Il n'en est pas de même pour les lithalses dont l'élévation est seulement de quelques mètres comme nous le montrons plus loin.

\section{LES lithalses allongÉs de BelgiQue}

Dans les Hautes Fagnes, certaines traces de lithalses sont étirées selon la pente et cette disposition a été expliquée pour la première fois par Muckenhausen (1960) par une alimentation préférentielle de la butte périglaciaire par des eaux arrivant dans le sol de l'amont (hypothèse reprise par Pissart dès 1963). Dans cette hypothèse, la croissance de ces lithalses allongées (la plus longue s'étend sur $800 \mathrm{~m}$, figure 6) s'est faite progressivement en s'étendant vers l'amont, vers la zone d'où arrivaient les eaux. Ce processus est une croissance latérale de lithalse dans une direction privilégiée.

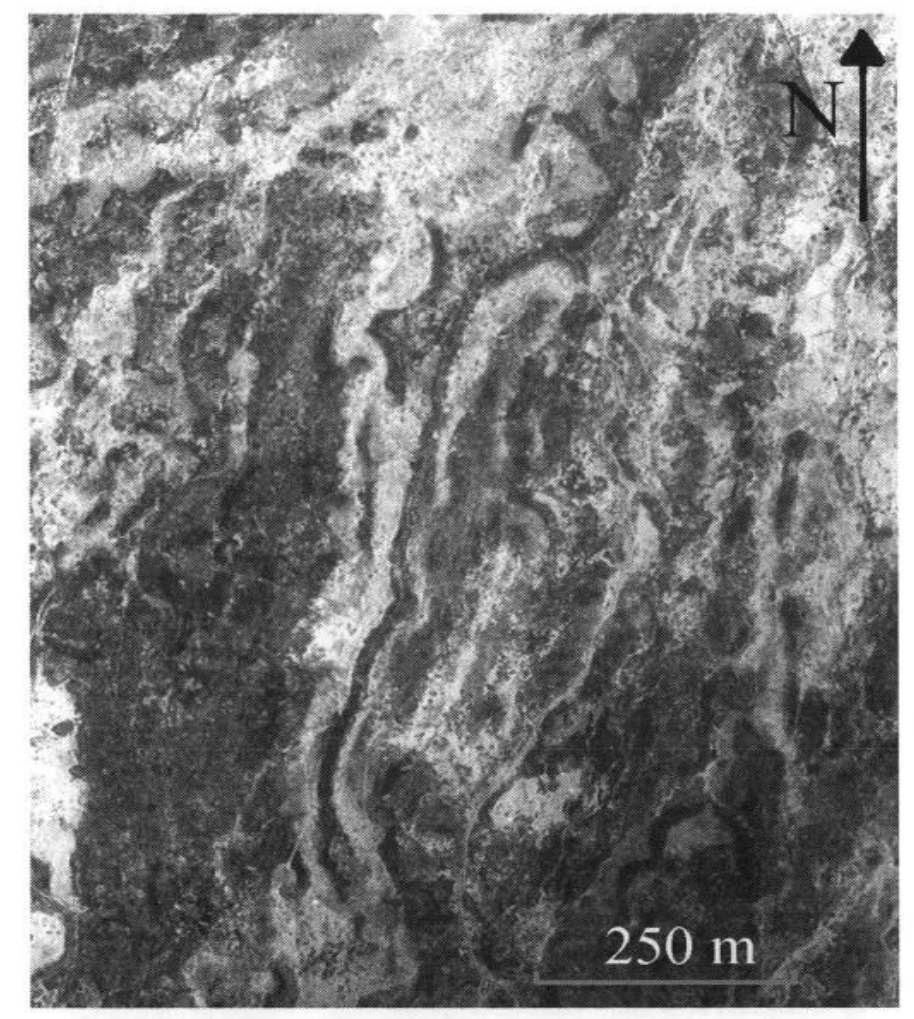

Fig. 6. - Photo aérienne de remparts de lithalses étirés selon la ligne de plus grande pente dans les Hautes Fagnes. Toutes les transitions existent entre des remparts circulaires et des remparts étirés selon la pente semblables à ceux que montre cette photo. 
LE TRANSFERT DES MATÉRIAUX DES LITHALSES VERS LES REMPARTS N'EST PAS SEULEMENT LE RÉSULTAT DE PROCESSUS DE VERSANTS

Dans un profil que nous avons réalisé au lieu dit La Brackvenn (figure 7, lithalse $\mathrm{X}$, localisation indiquée sur la figure 3 , Bastin et al., 1974) à $800 \mathrm{~m}$ de la coupe de la Konnerzvenn (figure 2), nous avons vérifié que le volume du rempart correspond bien au volume de la cuvette apparue au moment de la fusion de la glace.

Ce volume représente approximativement $3000 \mathrm{~m}^{3}$. Cela correspond à une cuvette dont la profondeur moyenne est un peu inférieure à $2 \mathrm{~m}$. Le problème qui se pose est de savoir comment cette masse de terre a été transférée en bordure de la lithalse.

Il est généralement admis, comme nous l'avons déjà dit, que pour les lithalses comme pour les pingos, les remparts sont apparus

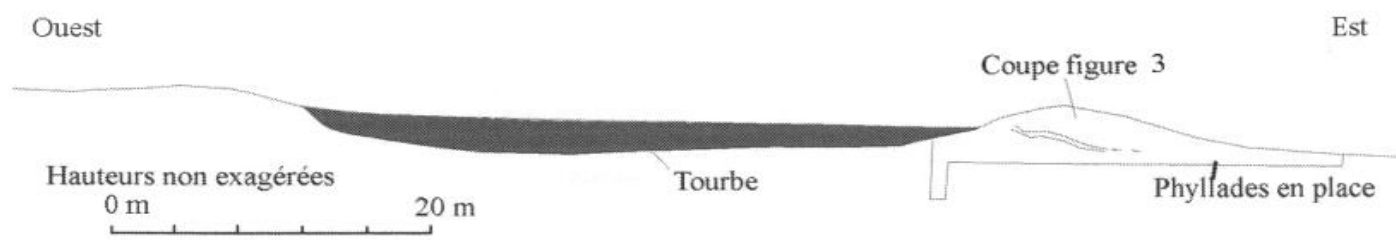

Fig. 7. - Profil au travers du vivier (trace de lithalse) désigné par la lettre $X$ sur la figure 4 et dont le rempart est décrit sur la figure 3 (Hautes Fagnes, Belgique). Le volume de la dépression en dessous de la surface existante avant la croissance de la lithalse correspond au volume de terre accumulée dans les remparts. La roche en place (phyllades très altérés) est visible à proximité, à moins de $2 \mathrm{~m}$ de profondeur.

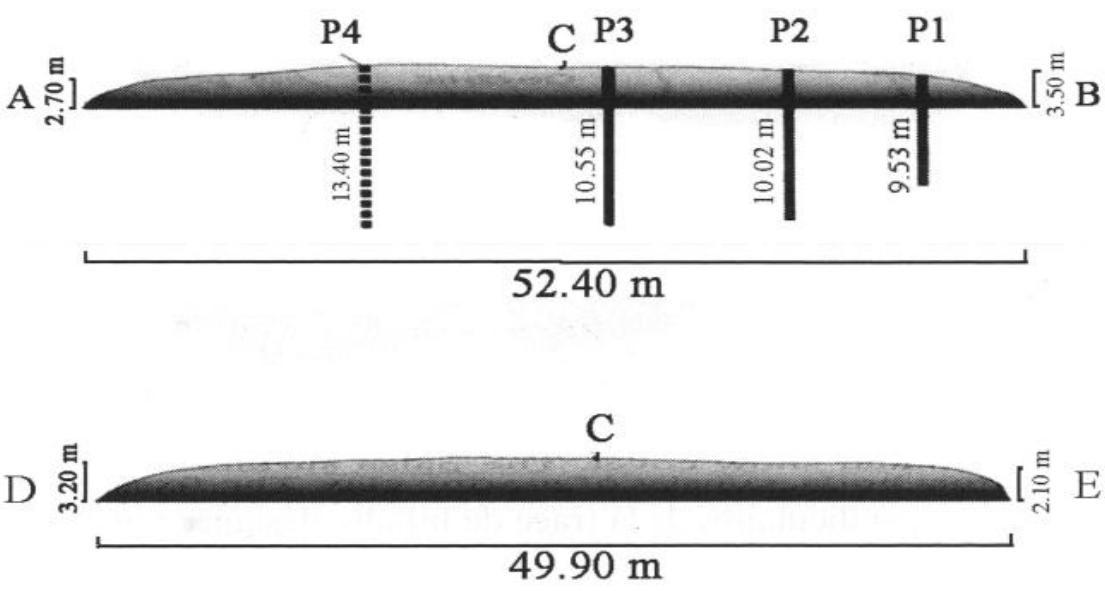

Fig. 8. - Profils perpendiculaires l'un à l'autre montrant la localisation des sondages dans la «BGR lithalsa en Hudsonie (figure 1). Les hauteurs ne sont pas exagérées. Les sondages atteignent la roche en place. 
par descente des matériaux sur les pentes de la butte périglaciaire. Toutefois, les lithalses actuelles telles qu'elles sont décrites au Québec sont toujours peu élevées et leurs surfaces sont presque horizontales. La hauteur maximale des lithalses en Hudsonie ne dépasse pas 7 mètres (communication orale de M. Allard en 2002). Il en résulte que le déplacement des matériaux par des processus de transport en masse ne peut se produire sur la lithalse. Ainsi, la lithalse étudiée par Calmels a $50 \mathrm{~m}$ de diamètre et $3.50 \mathrm{~m}$ d'élévation. Des pentes très accentuées n'existent qu'à la bordure des lithalses sur moins de $10 \mathrm{~m}$ de longueur (figure 8).

\section{LE PROFIL DES CUVETTES REMPlies de TOURBE DES HAUTES FaGNeS}

Nous ne connaissons actuellement que les profils de quelques cuvettes remplies de tourbe qui sont apparues lorsque la glace des lithalses a fondu. Nous savons toutefois que dans les Hautes Fagnes à côté de dépressions peu profondes comme celles que montrent les figures 7 et 9 , avec un fond plat et une profondeur moyenne de l'ordre de $2 \mathrm{~m}$ par rapport au niveau du sol originel, existent des cuvettes plus profondes atteignant presque $4 \mathrm{~m}$ de profondeur (figure 10) sous la surface «prélithalse» du sol et d'allure plus ou moins conique.

Pour expliquer la différence de forme des cuvettes, un élément essentiel est l'importance de la couche de limon qui permet la croissance de la glace de ségrégation. En 1974, nous avons publié les observations réalisées dans un sondage de $25 \mathrm{~m}$ de profondeur

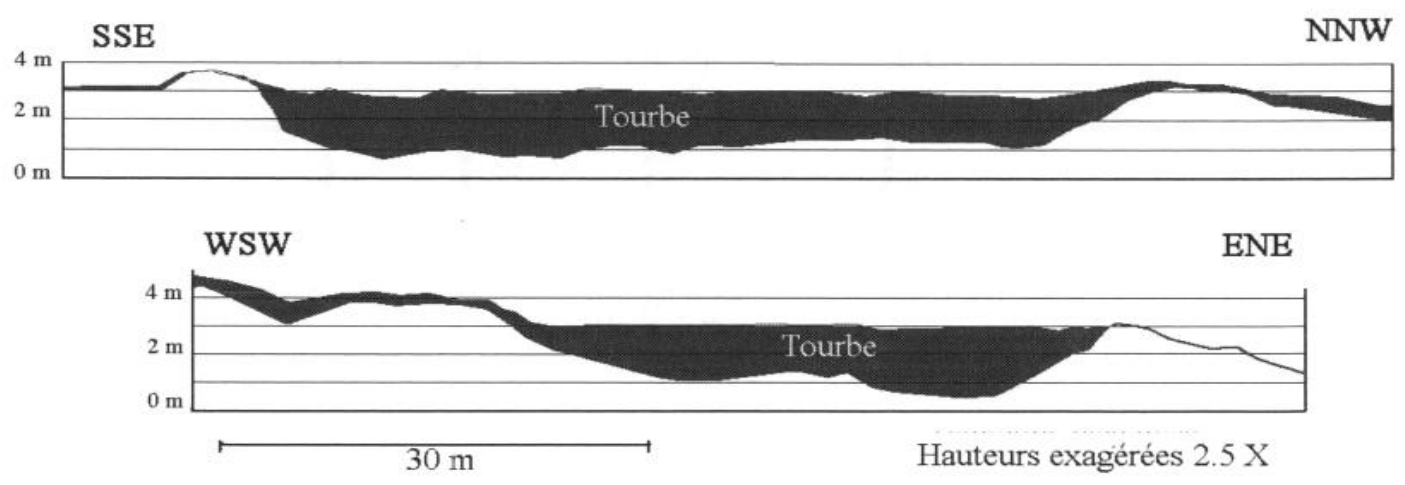

Fig. 9. - Profils perpendiculaires de la trace de lithalse désignée par la lettre Y sur la figure 4.

Cette trace de lithalse se trouve près des sondages 2 et 3 indiqués aussi sur la figure 4. Ces sondages attestent de la faible épaisseur des matériaux limoneux en cet endroit, ce qui explique vraisemblablement la faible profondeur de cette cuvette. 


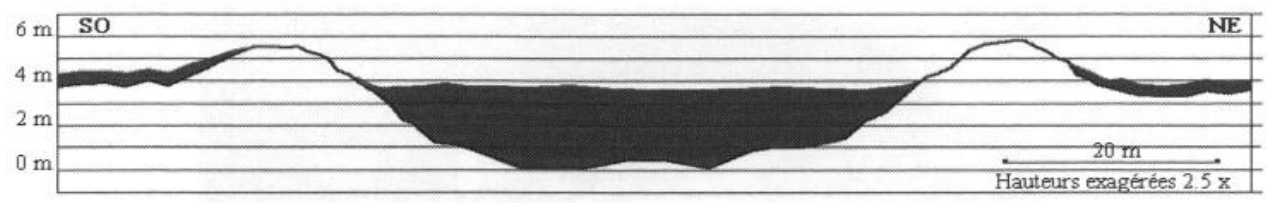

Fig. 10. - Profil d'une des traces de lithalse ( $Z$ sur la figure 11 ) partiellement vide de tourbe au lieu-dit la «Brackvenn» dans les Hautes Fagnes en Belgique

(Pissart, 1974) à la bordure immédiate de cette trace de lithalse (localisation: figure 4, sondage 2). Seulement $3 \mathrm{~m} 50$ de limon recouvrent en cet endroit le substratum cambrien fortement altéré. De $3,50 \mathrm{~m}$ à $11,05 \mathrm{~m}$, le sondage a traversé des argiles blanches avec des débris de quartzites et ce n'est qu'à partir de cette dernière profondeur que les quartzophyllades altérés sont reconnaissables. Des traces de cette altération qui est apparue avant le Crétacé (on est au niveau de la surface précrétacique) sont toujours visibles à $25 \mathrm{~m}$ de profondeur. L'argile blanche n'a jamais été observée dans les remparts et nous ne pensons pas que la glace de ségrégation s'y est beaucoup développée.

Le sondage 3 situé moins de $80 \mathrm{~m}$ au nord et à seulement $35 \mathrm{~m}$ de la même lithalse $Y$ (localisation: figure 4, chiffre 3) a montré la présence à $3 \mathrm{~m} 10$ de profondeur des phyllades noirs altérés en argile mais où la schistosité était toujours visible. Dans ce sondage, entre $2 \mathrm{~m} 60$ et $3 \mathrm{~m} \mathrm{10}$, les observations manquent, mais de la surface à $2 \mathrm{~m} 60$ se trouvent des limons très favorables à l'apparition de la glace de ségrégation.

Il est clair ici que la glace des traces de lithalse $\mathrm{X}$ et $\mathrm{Y}$ qui présentent un fond plat n'a pu apparaître que sur une épaisseur limitée de sédiments et donc que la hauteur de la lithalse a été peu importante. Il est donc très vraisemblable que cette lithalse avait une surface horizontale.

Par contre, des cuvettes plus profondes (figure 10, localisation sur la figure 11) se trouvent dans une zone où les formations limoneuses sont beaucoup plus épaisses. Un sondage de $23 \mathrm{~m}$ de profondeur réalisé à l'emplacement du parking Nasthief à environ $600 \mathrm{~m}$ au nord de la lithalse $Z$ de la figure 10 a montré qu'il y avait en cet endroit une épaisseur de $14 \mathrm{~m}$ de dépôts de pente limoneux (Pissart, 1974; Juvigné et Pissart, 1979). Il est certain que, la glace de ségrégation a pu se développer ici à une plus grande profondeur. Toutefois, nous n'osons guère avancer des suppositions concernant la hauteur qu'a atteint la lithalse. 


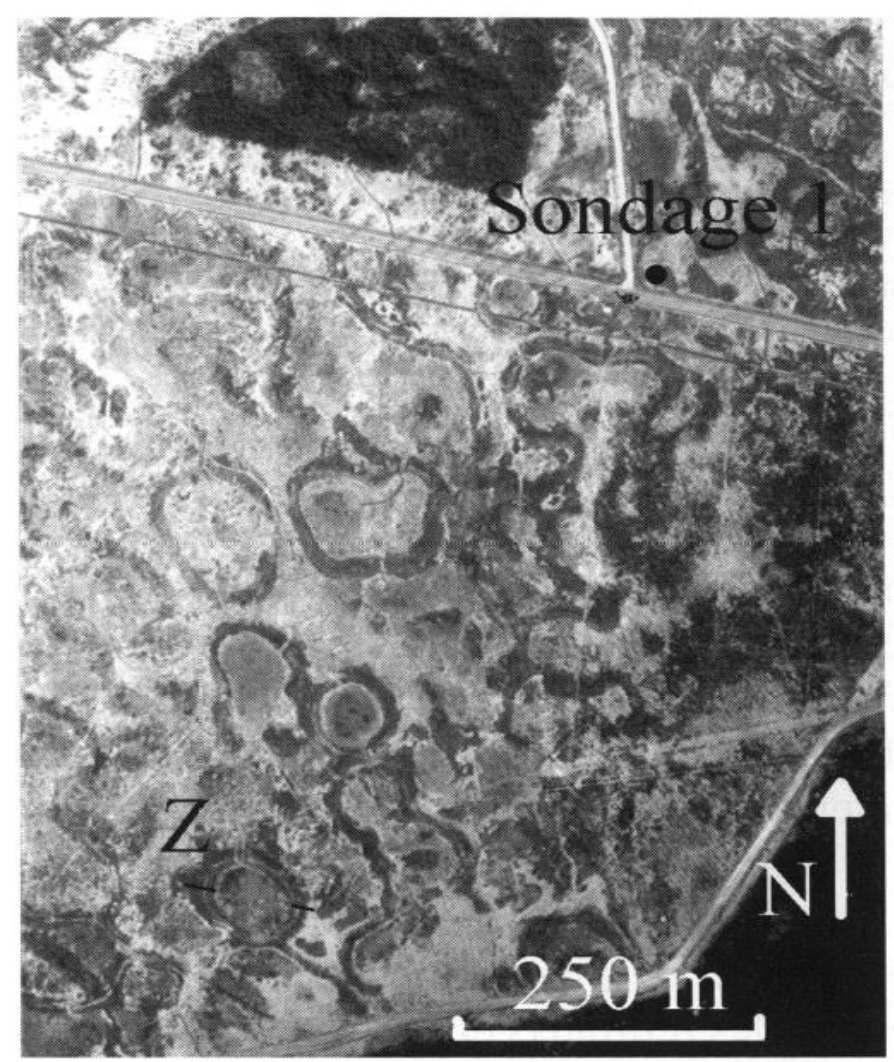

Fig. 11. - Photo aérienne montrant la localisation du vivier (trace de lithalse) $\mathbf{Z}$ dont le profil est donné sur la figure 10 (Hautes Fagnes, Belgique). Le sondage 1, décrit par Juvigné et Pissart (1979) a montré en cet endroit une accumulation de $14 \mathrm{~m}$ de matériaux limoneux favorables à l'apparition de glace de ségrégation.

Pour les lithalses dont les cuvettes ont le fond plat et dont la topographie de plateau était certainement semblable aux lithalses du Québec, il est impossible d'expliquer les remparts par le déplacement des matériaux de surface par des agents de transport en masse car d'un côté les pentes étaient trop faibles et d'autre part une érosion sur les lithalses aurait probablement déclenché des phénomènes thermokarstiques qui auraient déterminé, indépendamment de tout changement climatique, leur disparition.

\section{PENTES Fortes EXTÉRIEURES DES Lithalses}

Il existe en Hudsonie des formes de quelques mètres de diamètre et des formes ovales très régulières de plus de $70 \mathrm{~m}$ comme le montre la photo aérienne donnée par Calmels (figure 5). Les bordures des lithalses sont toujours en pente forte et les parties supérieures des lithalses presque horizontales. Les fortes pentes de bordure suggèrent un déplacement récent des remparts car elles 
devraient, vu leur forte inclinaison sous un climat aux fréquentes alternances gels-dégels, avoir subi une érosion amenant une réduction des pentes. Calmels souligne d'ailleurs plusieurs fois dans son travail la présence de nombreux «landslides» sur ces abrupts très raides. Il attribue ces glissements au réchauffement climatique dont il trouve des traces indiscutables dans l'affaissement thermokarstique d'une partie de la lithalse. Toutefois, comme le montrent les sondages, il n'y aurait pas sous les remparts un pergélisol dont la table pourrait, en reculant, expliquer le déclenchement de ces glissements. Nous n'y voyons donc pas l'effet du réchauffement mais seulement un processus, certes lié aux alternances de gel-dégel, mais sans liaison avec une fonte du pergélisol. C'est seulement un processus normal sur une pente aussi forte sous le climat de Hudsonie.

Dans les Hautes Fagnes, la pente extérieure des remparts de lithalse dépasse parfois $30^{\circ}$, ces pentes fortes dans des matériaux meubles ne peuvent pas provenir de l'accumulation de dépôts de pente mais résultent vraisemblablement d'une poussée profonde faisant reculer le rempart. Ces pentes ont été conservées en Belgique depuis l'Holocène étant donné que la couverture végétale a toujours protégé ces sites de l'érosion.

\section{LA DISPOSITION DES LITHALSES LES UNES PAR RAPPORT AUX AUTRES} EN HUDSONIE

La photo aérienne de 1957 publiée par Calmels (figure 5) montre des lithalses majoritairement séparées les unes des autres et dont la forme est le plus souvent régulière. La photo de Hudsonie que nous avons publiée en 2002 (Pissart, 2002) montre par contre des formes accolées qui suggèrent un développement latéral se poursuivant jusqu'au moment où les lithalses se rencontrent.

\section{EN HUdSONIE, LES MATÉRIAUX QUI FORMERONT LES REMPARTS (APRÈS LA FUSION DES LITHALSES) SONT ACCUMULÉS DANS UNE ZONE SANS PERGÉLISOL, EN BORDURE DE CES BUTTES PÉRIGLACIAIRES.}

Les photos aériennes des lithalses en fusion de Hudsonie montrent que les remparts des lithalses paraissent comparables en dimensions aux remparts des Hautes Fagnes. Un sondage décrit par Calmels permet de se rendre compte que le rempart potentiel 


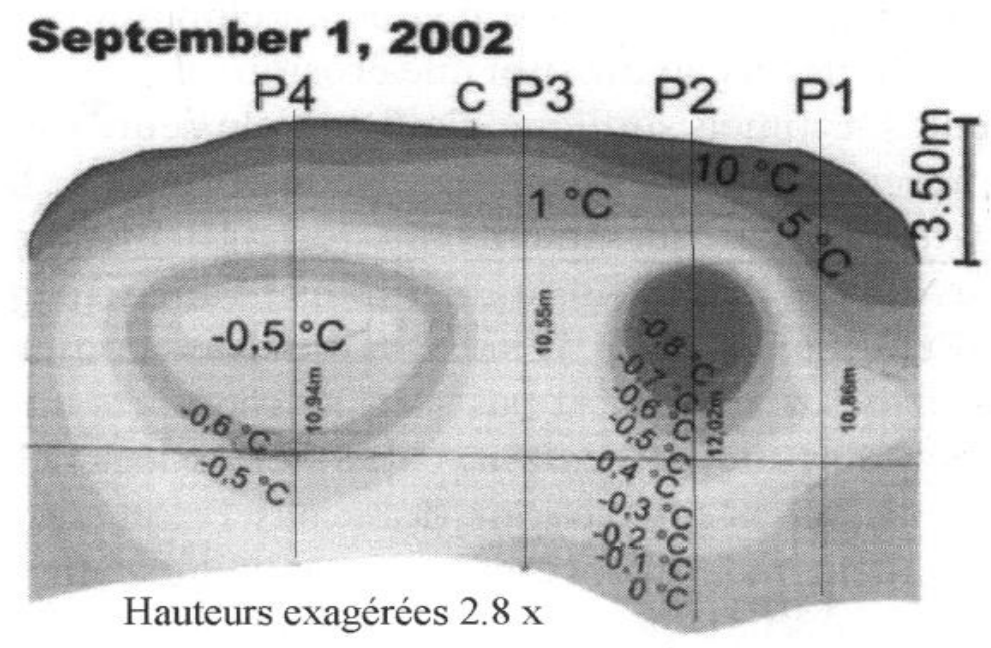

Fig. 12. - Fig. 4.8 de la thèse de Calmels, p. 71. Températures dans la lithalse de Hudsonie le $1^{\text {er }}$ septembre 2002 avant la reprise de la couche active par le gel. Les capteurs de température sont localisés uniquement dans les sondages. Aucune observation n'est recueillie à la bordure de la lithalse au-delà du sondage Pl et les tracés des isothermes y sont donc incertains. La précision des mesures traitées est de $0.2^{\circ} \mathrm{C}$ du fait des performances de l'enregistreur de données. Cette figure montre clairement que, s'il y a un pergélisol sous le sondage $\mathrm{P} 1$, il est en profondeur $(6 \mathrm{~m})$ et extrêmement limité.

(c'est-à-dire la matière minérale accumulée en bordure de la forme qu'il a étudiée) est important. En effet sur la partie de la figure que nous reproduisons (figure 13), le sondage $\mathrm{P} 1$ a été réalisé à $7 \mathrm{~m}$ de la pente abrupte qui constitue la limite de la lithalse (voir localisation sur la figure 8). Les mesures de température ont montré qu'il n'y avait pas dans ce sondage de sol gelé jusqu'à une profondeur de l'ordre de $6 \mathrm{~m}$ (figure 12), ce qui fait écrire à Calmels (p. 69) «que la section périphérique de la butte est à des températures légèrement supérieures ou légèrement inférieures à $0^{\circ} \mathrm{C} »$. Les températures observées au $1^{\text {er }}$ septembre 2002 indiquent que, dans le sondage $\mathrm{P} 1$, la partie qui serait sous $0^{\circ} \mathrm{C}$ est très réduite et qu'il ne peut y avoir que très peu de glace en cet endroit. A l'emplacement du sondage, la lithalse est donc constituée presque uniquement des matières minérales qui vont former le rempart. (La précision des mesures de température est de $0.2^{\circ} \mathrm{C}$ et le dessin des isothermes proposés par Calmels entre le sondage et la bordure de la lithalse n'est étayé par aucune observation en dehors du sondage luimême; cela ne met toutefois pas en cause les observations que nous avons mentionnées).

Les matériaux du rempart sont donc bien présents avant la fusion de la glace qui se trouve dans la lithalse et, pour cette 
lithalse, la hauteur du rempart qui subsistera est d'environ $75 \%$ de la hauteur du point le plus élevé de la lithalse.

Bien que la surface de la lithalse soit en très faible pente (légèrement convexe), Calmels estime qu'en l'absence de végétation, les matériaux des «frost boils» qui recouvrent la lithalse auraient pu être déplacés par les processus de surface vers les bordures pour former les remparts. Cette hypothèse rencontre une objection que nous avons mentionnée précédemment: si la surface de la lithalse était érodée, cela déclencherait par départ de la partie supérieure de la couche active, une fusion de la quantité de glace très importante qui se trouve immédiatement sous la couche active (moyenne de glace dans tout le profil, 65\% avec une teneur particulièrement importante immédiatement sous la table du pergélisol qui se trouve à $1.50 \mathrm{~m}$ de profondeur).

\section{LA POSITION OBLIQUE DES LENTILLES DE GLACE SUGGÈRE UN} ÉLARGISSEMENT PAR CROISSANCE LATÉRALE DU PERGÉLISOL.

Malheureusement, l'orientation des sondages réalisés dans la lithalse n'a pas été enregistrée. Par conséquent la direction des pendages des lentilles de glace n'est pas connue. Toutefois Calmels souligne (p. 64): "From the permafrost table to $2.5 \mathrm{~m}$," (soit sur une épaisseur de $1 \mathrm{~m}$ ) "most of the ice lenses are horizontal and vertical joints and faults are not abundant. From $2.5 \mathrm{~m}$ downward, the lenses have high dip angles". Comme les lentilles de glace apparaissent parallèlement à la direction du front de gel, cette observation suggère fortement que le gel a pénétré verticalement dans la lithalse à la partie supérieure de celle-ci mais obliquement à plus grande profondeur.

\section{Le mécanisme probable déterminant l'élargissement des lithalses}

D'après les observations de Calmels, les bordures actuelles des lithalses pourraient se présenter comme le montre la figure 13.

Le profil extérieur du rempart est celui dessiné par Calmels dans sa thèse. Il est identique à celui dessiné par Delisle et Allard (2003). L'échelle des hauteurs de cette figure n'est pas exagérée. Les températures considérées à l'emplacement du sondage P1 (figure 12) permettent de tracer obliquement la limite du pergélisol actuel par rapport à la surface du sol initiale. 


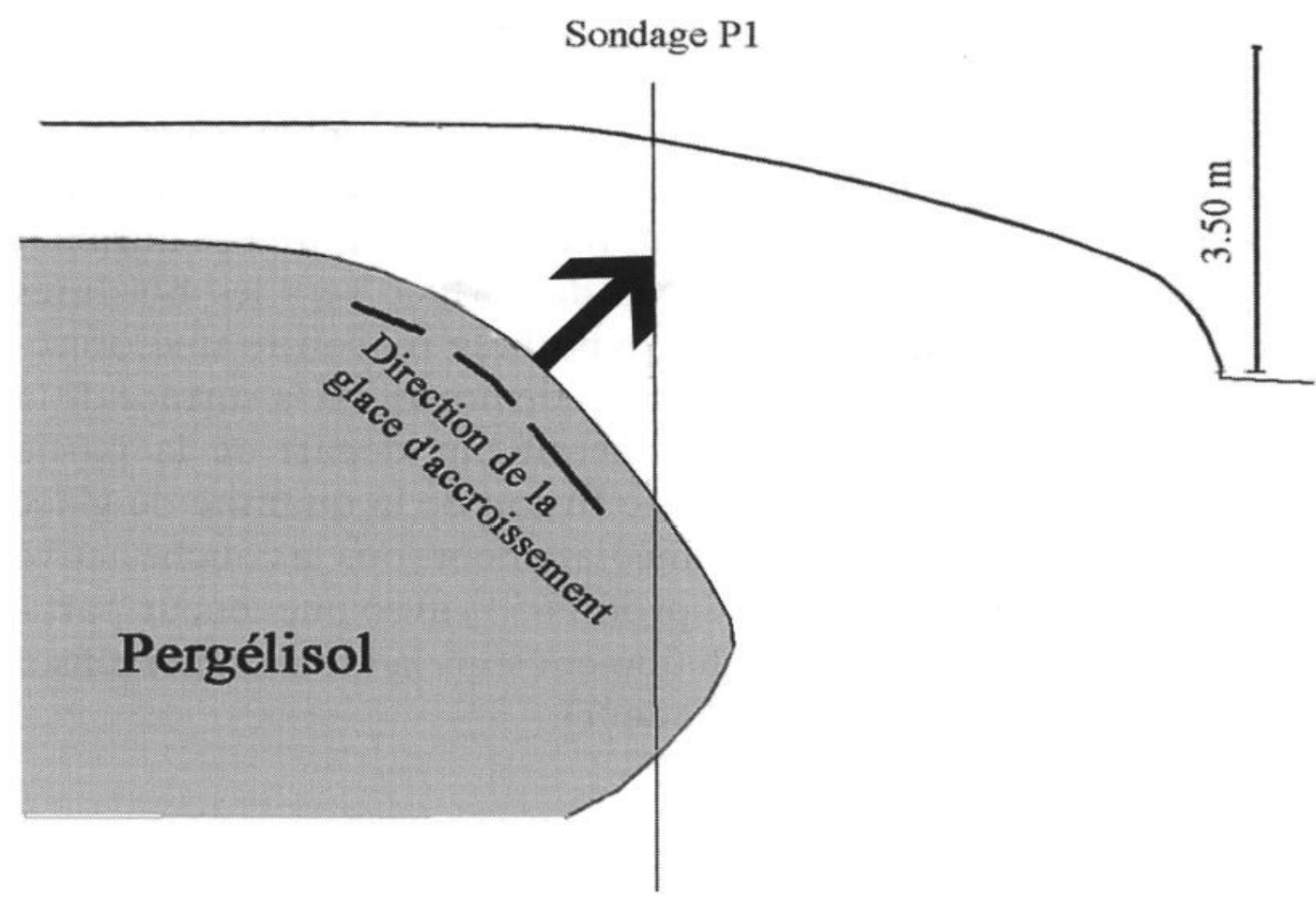

Fig. 13. - Mécanisme probable à l'origine de l'élargissement des lithalses. Le profil extérieur du rempart est celui dessiné par Calmels dans sa thèse. Il est identique à celui dessiné par Delisle et Allard (2003). L'échelle des hauteurs sur cette figure n'est pas exagérée. Les températures ont été observées à l'emplacement du sondage P1 (figure 12 extraite de Calmels p. 71). Elles permettent de proposer la limite du pergélisol. La glace d'accroissement qui apparaît en bordure du pergélisol est disposée parallèlement à la limite de celui-ci. Cette glace détermine une poussée dans les sédiments orientée selon la direction de la flèche.

Si la lithalse se développe, la glace qui va se développer sur cette partie inclinée de la table du pergélisol va provoquer une poussée qui sera perpendiculaire au front du pergélisol (figure 13). Ce processus bien décrit par Washburn $(1979$, p. 79$)$ sous le nom de «frost thrusting» a été très rarement invoqué dans le domaine de la géomorphologie périglaciaire. Le terme a été proposé par Eakin en 1916 (p. 76) qui n'a toutefois impliqué son action que dans l'évolution de sols polygonaux triés (Eakin, p. 80).

La pression de croissance maximum de cette glace de ségrégation est exprimée selon Williams (1981) par la formule

$$
\mathbf{P}^{\prime} i=\mathrm{P}^{\prime} w+(\mathrm{Pi}-\mathrm{Pw}) \mathrm{T}
$$

où $(\mathrm{Pi}-\mathrm{Pw}) \mathrm{T}$ est la succion qui est déterminée par la température T. Dans cette formule $P^{\prime} w$ est la pression de pore de l'eau disponible pour une éventuelle migration vers le front de gel. Washburn 
(1979, p. 69) donne les mêmes formules et précise que la pression suffisante pour arrêter la croissance de la glace de ségrégation s'appelle la «shut-off pressure», terme créé par MCRoberts and Nixon (1975, p. 42).

Williams souligne que la température qu'il convient d'appliquer est rarement connue et donc que les calculs de la pression sont aléatoires. Sans entrer dans aucun calcul, il est évident que dans les silts argileux de la lithalse étudiée, la présence de la glace de ségrégation jusqu'à une profondeur de $10 \mathrm{~m}$, montre que cette glace a développé une pression de 1.3 atmosphère, pression nécessaire pour soulever les sédiments et la glace qui la surmonte et cela, bien qu'à ce niveau, la température soit très peu inférieure à $0^{\circ} \mathrm{C}$.

Au sommet et en bordure de la lithalse, directement sous la couche active, les températures varient beaucoup au cours de l'année, aussi la pression peut être beaucoup plus élevée, de l'ordre de $10^{6} \mathrm{~N} \mathrm{~m}^{-2}$ (10 atmosphères) dans différents matériaux argileux à la température de $-0.5^{\circ} \mathrm{C}$ (Williams, 1981 , fig. 7.6). Cette pression développée par croissance de glace d'aggradation a, du fait de sa direction oblique, repoussé latéralement les matériaux donnant ainsi naissance au rempart.

Le professeur de mécanique des sols à l'Université de Liège, Albert Bolle, a calculé la poussée nécessaire pour déplacer les terres sur une épaisseur de $2 \mathrm{~m}$ selon la méthode de Rankine en admettant les cofficients suivants: un angle de frottement interne de $30^{\circ}$, une cohésion de $50 \mathrm{kPa}$ et une densité des terres de $2000 \mathrm{Kg}$ par $\mathrm{m}^{3}$. La poussée nécessaire pour une limite du pergélisol inclinée de $60^{\circ}$ et une profondeur de $1 \mathrm{~m}$ sous une surface horizontale est de $170 \mathrm{kPa}$ (1.7 atmosphère) pour déplacer les terres latéralement. À $2 \mathrm{~m}$ de profondeur, la poussée doit atteindre $230 \mathrm{KPa}$ (soit 2.3 atmosphères).

Ce processus de frost thrusting est fortement influencé par l'accumulation de neige en bordure de la lithalse. Il joue probablement un rôle dans des phénomènes liés à la nivation et par exemple dans la formation des grandes guirlandes qui affectent les replats de cryoturbation que nous avons décrits au Pays de Galles (Pissart, 1963).

Au moment du regel, quand apparaît la plus grande partie de la glace d'aggradation, la température est proche de $0^{\circ} \mathrm{C}$. Mais nous savons bien que toute l'eau ne gèle pas à $0^{\circ} \mathrm{C}$ et qu'une quantité d'eau non négligeable subsiste à une température inférieure, eau qui va alimenter la glace de ségrégation à des températures 
plus basses en dégageant des pressions importantes. Même si cette quantité d'eau est minime, si le phénomène se reproduit pendant plus de 1000 ans, le déplacement qui en résulte n'est certainement pas négligeable.

Il reste toutefois à expliquer comment dans les deux coupes que nous avons étudiées dans les Hautes Fagnes et qui se trouvent à l'aval de formes allongées, 77 et $62 \%$ de la masse des remparts sont constitués de dépôts de pente. Il est possible que dans ces deux coupes, les processus de versants ont remanié les sédiments repoussés par la croissance de la glace et arrivant en haut de l'abrupt et que ces dépôts aient stabilisés les remparts.
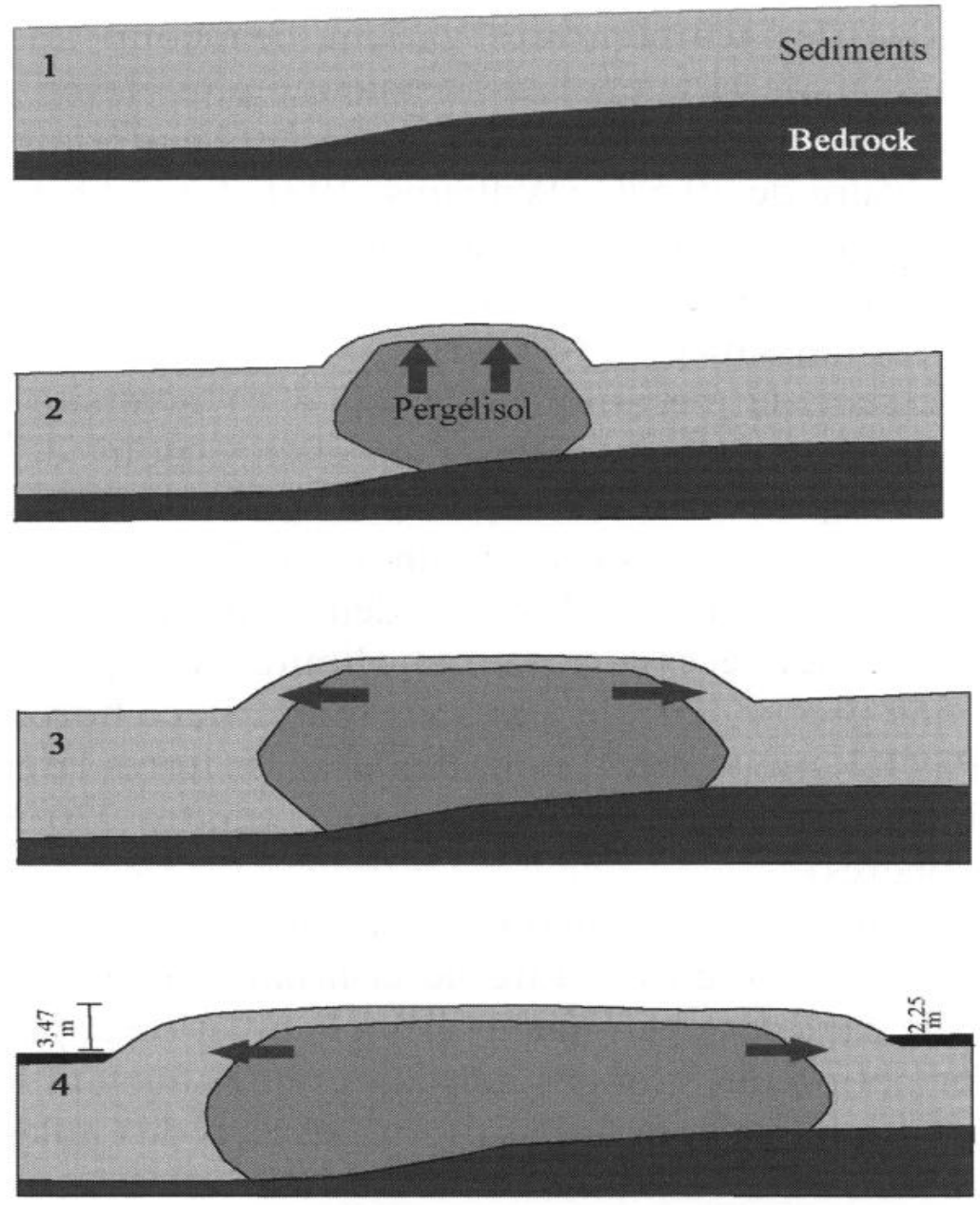

Fig. 14. - Schéma montrant la formation de la lithalse étudiée par Calmels selon l'hypothèse défendue dans le présent article. Les hauteurs ne sont pas exagérées. Noter l'accroissement des volumes de matériaux formant les remparts en même temps que l'élargissement de la lithalse (figure 4.12 modifiée de la thèse de Calmels, p. 108). 
Les observations de Calmels ne permettent pas de vérifier cette hypothèse du déplacement des matériaux. Les quantités de glace qu'il a mesurées à savoir $65 \%$ de glace, si elles se poursuivent jusqu'à $1 \mathrm{~m}$ de la roche en place soit sur une épaisseur de $7.50 \mathrm{~m}$ (en décomptant la couche active et la zone sableuse de la base) doit amener un soulèvement de près de $5 \mathrm{~m}$ et plus là où les argiles silteuses ont $10 \mathrm{~m}$ d'épaisseur. Comme le soulèvement maximum mesuré sur le terrain est de $3.50 \mathrm{~m}$, il manque $1.50 \mathrm{~m}$ de sédiments (moins le retrait de l'argile silteuse suite à son assèchement entre les lentilles de glace de ségrégation). Cette différence entre le soulèvement calculé et le soulèvement observé peut s'expliquer de deux façons, soit la teneur en glace diminue avec la profondeur, soit il y a eu effectivement déplacement des matériaux. Bien que les deux explications puissent être invoquées ensemble, étant donné que les lithalses laissent après leur fusion des dépressions entourées de remparts, on doit admettre qu'une quantité de sédiment a été déplacée comme le propose d'ailleurs Calmels. Il pense toutefois qu'il y avait au départ de la tourbe au sommet de la lithalse, ce qui ne paraît pas prouvé.

En conséquence le schéma de la croissance des lithalses généralement admis (Pissart, 2002; Calmels, 2005) pourrait être modifié comme nous le proposons sur la figure 14 .

\section{Conclusions}

Des arguments nombreux provenant des traces de lithalses de Belgique ainsi que d'autres issus des recherches réalisées par l'équipe de Michel Allard en Hudsonie et rapportées en détail par F. Calmels, permettent de proposer que les lithalses s'étendent par croissance latérale et donc que les schémas de croissance des lithalses proposés jusqu'ici doivent être modifiés. Le mécanisme en cause, lié à l'inclinaison de la table du pergélisol en bordure des lithalses, est relativement peu connu: il est dénommé «frost thrusting».

Les propositions du présent article pourront être aisément vérifiées ou contredites en excavant des coupes à la bordure des lithalses. Celles-ci sont facilement réalisables en utilisant une pompe portative comme l'ont montré nos recherches dans des pingos (Pissart, 1967; Pissart et French, 1976) et même dans une lithalse (Pissart et Gangloff, 1984). La lithalse que nous avons excavée était de dimension réduite et en fusion de telle sorte que 
nous n'avons pas pu observer la structure du rempart. Les coupes à réaliser en Hudsonie seront aisées étant donné la granulométrie fine des matériaux dans lesquels se sont développés les lithalses et qui, de ce fait, seront transportées facilement par l'écoulement des eaux projetées par la pompe.

\section{Remerciements}

Je prie le Professeur de mécanique des sols A. Bolle de l'Université de Liège d'accepter mes remerciements pour avoir bien voulu calculer la force développée par la glace de ségrégation pour repousser des terres vers le rempart des lithalses. Alain Demoulin a relu le manuscrit et a suggéré de nombreuses améliorations. Je lui en suis reconnaissant. Je remercie aussi mes collègues Jean Grimbérieux et Geoffrey Houbrechts ainsi que mon petit fils Renaud Pissart pour l'aide qu'ils m'ont accordée lors de levés de terrain.

\section{Bibliographie}

Allard, M., Caron, S. and Begin, Y., 1996. Climatic and ecological controls on ice segregation and thermokarst: the case history of a permafrost plateau in Northern Québec. Permafrost and Periglacial processes, 7, 207-227.

Bastin, B., Juvigné, E., Pissart, A. et Thorez, J., 1974. Etude d'une coupe dégagée à travers un rempart d'une cicatrice de pingo de la Brackvenn. Annales de la Société géologique de Belgique, 97, 341-348.

Buteau, S., Fortier, R., Delisle, G. et Allard, M., 2004. Numerical simulation of the impacts of climate warming on a permafrost mound. Permafrost and Periglacial Processes, 15: 41-57.

Calmels, F., 2005. Genèse et structure du pergélisol. Etude des formes périglaciaires de soulèvement au gel au Nunavik (Québec Nordique au Canada). Thèse de doctorat présentée à l'Université de Caen, $169 \mathrm{p}$.

Calmels, F. et Allard, M., 2004. Ice segregation and gas distribution in permafrost using tomodensimetry analysis. Permafrost and Periglacial Processes, $15:$ 367-378.

Calmels, F., Gagnon, O. et Allard, M. 2005. A portable earth-drill system for permafrost studies. Permafrost and Periglacial Processes, 16: 311-315.

Calmels, F. and Allard, M., 2007a. A structural interpretation of the palsa/lithalsa growth mechanism through the use of CT Scanning. Farth Surface Processes and Landforms, in press. (Published online 20 June 2007 in Wiley InterScience). 
Calmels, F., Allard, M. and Delisle G., 2007b. Development and decay of a lithalsa (Northern Québec): A geomorphological history. Geomorphology, in press. (published online 1 September 2007 in DirectScience).

Calmels, F., Delisle G. and Allard, M. 2007c. Internal structure and the thermal/hydrological regime of a lithalsa: significance for permafrost growth and decay. Canadian Journal of Earth Sciences, in press. Accepted 5 September 2007.

Delisle G. et Allard M., 2003. Numerical simulation of the ground température field in a palsa reveals strong influence of convective heat transport by groundwater. Permafrost. Proceedings of the eighth international conference on permafrost, 21-25 July 2003, Zurich, Switzerland, Balkema Publishers:181-186.

Delisle G., Allard M., Fortier, R., Calmels, F. et larrivée, E., 2003. Umiaujaq, Northern Québec: innovative techniques to monitor the decay of a lithalsa in response to climate change. Permafrost and Periglacial processes, 14 : 375-385.

Juvigné, E. et Pissart, A., 1979. Un sondage sur le plateau des Hautes Fagnes au lieu dit la Brackvenn. Annales de la Société géologique de Belgique, 102: 277-284.

Mackay, J. R., 1979. Pingos of the Tuktoyaktuk peninsula area, Northwest Territories. Geographie physique et Quaternaire, 33 (1): 3-61.

MCRoberts, E. C. and Nixon, J. F., 1975. Some geotechnical observations on the role of surcharge pressure in soil freezing. Am., Geophys. Union, Conference on Soil-Water Problems in Cold Regions (Calgary, Alberta, Canada, 6-7 May 1975), Proceedings: 42-57.

Muckenhausen, E., 1960. Eine besondere Art von Pingos am Hohen Venn/ Eifel. Eiszeitalter und Gegenwart, $11: 5-11$.

PIsSART, A., 1956. L'origine périglaciaire des viviers des Hautes Fagnes. Annales de la Société géologique de Belgique, 79: 119-131.

Pissart A., 1963. Origine périglaciaire d'une variété géante de sols en guirlandes découverte au Pays de Galles. Compte Rendu de l'Académie des Sciences, Paris. 256: 222-224.

Pissart, A., 1963. Les traces de «pingos» du Pays de Galles (Grande Bretagne) et du plateau des Hautes Fagnes (Belgique). Zeitschrift für Geomorphologie, 7 (2): 147-165.

PISSART, A., 1963. Des replats de cryoturbation au Pays de Galles (Une variété géante de sols en guirlandes). Biuletyn Peryglacjalny, 12: 119-135.

Pissart, A., 1967. Les pingos de l'Ile Prince Patrick. Geographical Bulletin, 9 (3) : $189-217$.

Pissart, A., 1974. Les viviers des Hautes Fagnes sont des traces de buttes périglaciaires. Mais s'agissait-il réellement de pingos. Annales de la Société Géologique de Belgique, 97 : 359-381.

Pissart, A., et French, H., 1976. Pingo investigations, north-central Banks Island, Canadian Arctic. Canadian Journal of Earth Sciences, 13 (7): 937-946.

Pissart, A. ct Gangloff, P., 1984. Les palses minérales et organiques de la vallée de l'Aveneau, près de Kuujjuaq, Québec sub-arctique. Géographie physique et Quaternaire, 38 (3): 217-228. 
Pissart, A. et JuvignÉ, E., 1980. Genèse et âge d'une trace de butte périglaciaire (pingo ou palse) de la Konnerzvenn (Hautes Fagnes, Belgique). Annales de la Société géologique de Belgique, 103: 73-86.

Pissart, A., 1987. Géomorphologie Périglaciaire - Texte des leçons de la Chaire Francqui belge 1987. Edition du laboratoire de Géomorphologie et Géologie du Quaternaire de l'Université de Liège, 135p.

Pissart, A., 1998. Les traces des buttes périglaciaires des Hautes Fagnes et le climat du Dernier Dryas (13.000 à 11450 ans avant aujourd'hui). Bulletin de la classe des Sciences de l'Académie royale de Belgique, $6^{\mathrm{e}}$ série, Tome IX, 7 (12): 403-437.

Pissart, A., 2000. The remnants of lithalsas of the Hautes Fagnes (Belgium): a summary of present day knowledge. Permafrost and Periglacial Processes, 11 (4): 327-355.

Pissart, A., 2002. Palsas, lithalsas and remnants of these periglacial mounds. A progress report. Progress in Physical Geography, 26 (4): 605-621.

Pissart, A., 2003. The remnants of younger Dryas lithalsas on the Hautes Fagnes Plateau in Belgium and elsewhere in the world. Geomorphology, 52:5-38.

Washburn, A. L., 1979. Geocryology. A survey of periglacial processes and environments. Edit. Edward. Arnold, London, 406 p.

Williams, P. J., 1981. The surface of the earth: an introduction to geotechnical science. Longman, London and New-york, $211 \mathrm{p}$. 